\title{
REVIEW
}

\section{Functional Expansion of Music Education Technologies (through the example of music rehabilitation therapy of neurology ward patients)}

\author{
Tatiana Vladimirovna Lvova ${ }^{1}$ Alla Vladimirovna Toropova ${ }^{2 *}$ \\ 1. Federal medical Biophysics Center named after A. I. Burnazyan, Russian Federation \\ 2. Moscow Pedagogical State University (MPSU), Russian Federation
}

\section{ARTICLE INFO}

Article history:

Received: 7 January 2018

Accepted: 10 January 2019

Published Online: 30 April 2019

Keywords:

Music psychotherapy

Parkinson's disease

Movement disorder

\section{ABSTRACT}

This article presents a research of the possibility to apply music education activities for rehabilitation of patients with neurodegenerative disease. The purpose of this work was development of a music psychotherapy program for rehabilitation of patients with Parkinson's neurodegenerative disease. We suggested "target objects" for music rehabilitation therapy at all levels of manifestation of symptoms of illness: 1) physical restriction and rigidness which includes well-aimed work with breathing and movement symptoms, loss of control, lack of coordination, inflexibility, tremor; 2) emotional deprivation (realization and expression of emotions and feelings) through plastics of movements to music and singing-seeking catharsis through vivified contact with oneself and intensification of feelings; 3)social deprivation: wellaimed work on resocialization of the patient. Results we obtained contribute to further understanding and wider application of music education methods, technologies and approaches that may add to rehabilitation practices.

\section{Introduction}

$\mathrm{T}$ The modern world has come to understanding that music as a psychotherapeutic tool in addition to treatment of emotional disorders can be a powerful correctional remedy capable to consistently decrease and in some case completely eliminate various types of so called somatic symptoms, effectively correcting the patient's condition and contributing to his or her rehabilitation in general. Currently, functional rethinking of music education methods and adaptation of music-related activities to fit medical or social-psychological purposes are critical questions which inspire researchers to explore new forms of rehabilitation programs among available technologies of music education.

\section{Theoretical Grounds}

This article presents a research of the possibility to apply music in therapy for rehabilitation of patients with Parkinson's neurodegenerative disease. Parkinsonism or Parkinson's disease is a chronic progressive disease of the brain that was characterized by an English doctor, James Parkinson. It is worth noting that formerly this disease was registered in patients of the age of over 60 . Now we observe this disease in patients younger than 50 years. Unfortunately, symptoms and manifestations of this disease intensify and worsen with time. Therefore, we believe that it is necessary to find therapy methods for early prevention, possible inhibition of development of the disease and provision of prolonged rehabilitation aid at a later stage.

\footnotetext{
*Corresponding Author:

Alla Vladimirovna Toropova

Moscow Pedagogical State University (MPSU), Russian Federation

Email: allatoropova@list.ru
} 
Application of music therapy approaches and other art technologies as therapy for Parkinson's disease has its own history. A program of dancing classes for people with Parkinson's disease, their friends and families, has been successfully developed since 2001 based on cooperation between the Mark Morris Dance Group (MMDG) and the Brooklyn Parkinson Group (BPG) ${ }^{1}$. Alex Kerten (2016) ${ }^{[1]}$, an Israeli therapist, is the ideological inspire of a gyro-kinetic therapy or dance therapy. In his work he combined music and certain movements that have a beneficial effect for the damaged human nervous system. Fellow researchers from the United States Stegemöller et al. $(2016 ; 2017)^{[2,3]}$ demonstrated an improvement of the swallowing function as a result of choir singing of patients with Parkinson's disease. A review from British researches Barnish et al. (2016) ${ }^{[4]}$ also mentions that singing is beneficial as part of rehabilitation therapy for Parkinson's disease.

Nevertheless, the potential of music therapy for rehabilitation of people with Parkinson's disease is far from being depleted.

The purpose of this work is finding theoretical grounds and development of a music therapy program for rehabilitation of patients with Parkinson's neurodegenerative disease.

Main manifestations of Parkinsonism are the following:

1) With time movements become slowed and constrained; as a rule this process begins from the right hand, resulting in difficulties with writing and performance of everyday activities, and later expands to the entire right side of the body, and as years go by, expands to the left side of the body as well;

2) all muscles are constantly strained (hypertonia), which leads to development of so called "posture of a beggar": bowed back, arms and legs flexed in joints, which also leads to development of chronic pain;

3) frozen facial expression and rare blinking create an illusion that the patient is angry or completely lacks emotions;

4) speech becomes unclear, a chance that hypersalivation can develop;

5) thinking is slowed, attention is weakened, but memory and intellectual abilities remain unchanged at initial stages;

6) about $50 \%$ experience decreased motivation and lose interest in what surrounds them, their general mood fades and chronic depression develops.

All such symptoms result not only in limited movement but also in drastic limitation of the circle of contacts and the range of interests. Therefore, there is no doubt that people with such a disease require aid that will include special activities aimed at resocialization, improvement of mental state, increase of general resistibility, increase in motivation to perform exercises and communicate with other people, as well as partial recovery and possible enhancement of movement plastics and coordination, recovery of the breathing function, etc.

Having analyzed main symptoms of this disease, we suggested "target objects" for music rehabilitation therapy at all levels of manifestation of symptoms of illness:

(1) physical restriction and rigidness which includes well-aimed work with breathing and movement symptoms-hypertonia, loss of control, lack of coordination, inflexibility, tremor;

(2) emotional deprivation (realization and expression of emotions and feelings) through plastics of movements to music and singing-seeking catharsis through vivified contact with oneself and intensification of feelings;

(3) social deprivation: well-aimed work on resocialization of the patient (change of the rigid mindset of keeping the painful symptoms through blocking of any communication and increasing isolation to a positive mindset of restoration of means of communication "as if" there is no hindering problem (illness)-this is an application of a stratagem developed by Giorgio Nardone in his brief strategic psychotherapy (2006) ${ }^{[5]}$, which gives an essential psychotherapeutic effect-emotionally corrective experience which in its turn is self-supporting strategy of breaking the isolation.

So let us review in detail capabilities of music rehabilitation effect on all levels of symptom manifestations.

Restoration of free breathing has crucial importance in music rehabilitation therapy. Vocal and breathing exercises help to enhance flexibility of the rib cage and diaphragm, remove strain in the respiratory muscles, increase expandability of the lungs, optimize gas exchange and correct respiratory insufficiency. Through breathing exercises (with and without singing), we strive for achieving positive emotions, from the inner calmness to the external one and vice versa. During such training it becomes possible to gain control of and regulate breathing in accord with plastic movements to music which we will refer to below.

It is well known that such patients suffer progressive change and weakening of their voice strength. They, as a rule, speak in a very small voice and unemotionally.

Introduction of singing is an important method in the music corrective therapy. Through singing we, unwittingly for the patient, train depth, rhythm, tempo and frequency of breathing. Such training gradually affects not only functions of the throat, neck muscles, lungs and diaphragm, but basically of the whole body.

Speech of people suffering Parkinsonism can be poorly 
modulated and unclear. The voice loses tones and becomes monotonous and weak. That is why we ask our patient to perform various types of such an exercise as speaking out a text or singing out individual words in various combinations, and after that the patients sing a few verses of a song they know or have learned.

As part of such exercises we set a number of goals:

1) Certain training of facial muscles. (It is common knowledge that facial gestures of many patients become less emotional. In some cases their face freezes in a stable expression of a focused and intense attention with an unwinking stare, the face can look like a mask). Active singing helps activate facial and extraocular muscles.

2) Training of throat muscles the main functions of which are vocalizing, protective and respiratory.

3) Articulatory exercises: we speak out words in an exaggerated manner involving facial muscles; in a very small voice, then louder, very loudly as from the stage and finally singing them out like an opera singer. Later this exercise makes patients smile, quickly cheers them up and makes them tune in to the work. This exercise already prepares for further singing because it warms up cords and prepares them for work.

4) These exercises start progressive work on memory training, because we ask patients to recall their favorite songs which will be used for further work in that direction.

Rehabilitation classes imply hard working to improve coordination of hand movements, general plastics, finding out and working with organs involved in manifestations of tremor, working on the vocal apparatus.

Such work includes exercises to enhance coordination of hand and foot movements and generally coordination of hands, feet, head and vocal apparatus (coping with synkinesis, i.e. generalized strains and spasms).

The following symptoms we put into one big group: it includes tremor, slowness of movements (bradykinesia), loss of automatism of movements, coordination disorders and loss of balance.

Symptoms of minor shaking of hands or individual fingers and tremor of lower limbs can be more expressed on one side of the body or may be expresses symmetrically and be combined with other symptoms of damage of the nervous system: coordination disorder, sensibility disorder, etc.

Tremor is especially expressed when a patient is in a state of stress. During practical lessons and personal interviews we pay great attention to stressful, uncomfortable for the patient, situations and teach how to reach relaxation through breathing and vocal exercises in order to get relieved from such emotions. This includes both a variety of exercises intended to develop deep breathing and singing out a sound with intentional relaxation of the body. During the first meeting we always ask the patient the following question: "When did you feel tremor for the first time?" Step by step, with the patient's help, we find out what, in the patient's opinion, was the predecessor of development of his or her disease and where (in which organ) the patient felt weakness or tremor (or other symptoms) for the first time. We want to emphasize that in such a way we select a target object for systematic treatment. At later stages of our therapy we introduce certain exercises according to previously obtained information, with obligatory involvement of the selected organ. (We do not conduct any psychoanalysis, do not put the patient in the psychological reliving of onset of the disease, but by means of plastics of movements, certain singing techniques and methods of relaxation through breathing and sounds of music we begin working out the symptom, so called "onset", the trigger zone.

The second, mandatory, series of questions during the first interview is: "What songs do you like? What songs from your childhood do you remember? What songs did you or your mother use to sing?" etc. Thus we can find music material we will be able to work with in future. What is the most important in this respect in our opinion? First: songs from childhood remain, as a rule, more environmental, intimate and "merciful" for a patient, lacking traumatic associations. Second: the patient quickly recalls words and thus intensifies the work of his or her brain; third: this leads to relaxation of the patient.

If a patient quickly recalls a childhood song, it also becomes helpful for working with physical coordination.

Patients learn to:

1) coordinate movements of hands with breathing before singing and during the exhale;

2) neurocorrectional exercises are introduced at each training session to develop or strengthen interhemispheric links and functional zones related to fine motor skills and holistic image thinking through smoothing of scaled-up movements, training of the speech apparatus, as well as after each relaxation exercise.

Singing and plastic movements suit perfectly for treating such patients. For example: when patients are singing, we teach them to move their hands in the following order: two hands, then the right hand (the left hand remains still), then the left hand (while the right hand remains still) and after that both hands again, etc.

Hand movements depend on the song timing so variations are possible. We consistently work to achieve measured and smooth hand movements and for that purpose all songs are sung in a slower tempo. At a later stage we 
try to sing while performing the hand exercise and introduce alternating foot stamping.

\section{Results of the Experiment}

27 patients participated in this pilot research, but conditions of our working with them were different: only 7 patients spent over 2 weeks in the ward, while 12 patients stayed there for two weeks and 8 patients spent less than two weeks there. Lessons were taking place 3 times a week, so the patients attended music rehabilitation lessons from 4 to 9 times in total.

In this experiment we could observe improvements not only in expression of external symptoms but also in cognitive functions as soon as by the 3rd lesson. We can say that such cognitive functions as Memory, Gnosis, Speech, Praxis and Intellect receive support and training during music education activities.

During first two lessons the patients had hard time with remembering and following the program of their task, but by the third lessons most of them were able to quite quickly understand which task they were asked to perform and performed exercises much quicker and more accurately. This shows that the function of perception of information, its subsequent processing and merging of sensory impressions into holistic images were beginning to get involved, which is an indicator of development of the posterior parts of the brain (temporal, parietal and occipital lobes).

By the third lessons some patients began to recall quite hard tasks on their own, perform them more consciously and with increased interest. The memory function was beginning to gradually expand and the patient was beginning to keep information more reliably. (It is known that the memory function is connected to activities of the entire brain, but the hippocampal structures have particular importance for remembering of current information, which we could observe in that particular case).

As soon as by the third lesson, speech of most patients became clearer, voice power and intelligibility gradually increased (singing, warm up, speaking out the text, slow tempo).

Also by the third lesson we could observe an improvement of Praxis. Patients were learning, remembering and later reproducing new trained movement skills based on plastic movements to music and complex interrelated and mutually coordinated hand and foot movements always accompanied by singing and speaking out rhythmical phrases. In such a way, both frontal and parietal lobes of the brain were involved in the process. Step by step, movement tasks during such lessons were becoming more complex. New coordination training tasks were added that aided to expand movement programs for the patient.
It is an incontrovertible fact that development of a certain music therapy complex depends on individual specificities of patients, their readiness for rehabilitation and the dynamic picture of changes in the condition of each patient. Thus, music rehabilitation activities have a great potential as therapy for Parkinson's disease.

\section{Conclusion}

1) This pilot experiment demonstrated that the suggested music rehabilitation program covers all levels of manifestations of symptoms in patients suffering such a neurodegenerative disorder as Parkinson's disease.

a) physical restriction and rigidness, spasticity, loss of control, loss of coordination, inflexibility, tremor;

b) emotional deprivations;

c) social deprivation;

2) The results we obtained contribute to further understanding and wider application of functions of music education methods, technologies and approaches that may add to rehabilitation practices.

\section{Footnote}

1 http://markmorrisdancegroup.org/community/Dancefor-PD/Dance-for-PD

\section{Acknowledgement}

We thank the Federal Medical Biophysical Center named after A.I. Burnazyan, Moscow for the opportunity to approbate the experimental musical psycho-therapeutic program and its discussion at a medical conference.

\section{Reference}

[1] Kerten, Al., Brinn, D. Goodbye Parkinson's, Hello life!: The Gyro-Kinetic Method for Eliminating Symptoms and Reclaiming Your Good Health. 2016, Divine Art. 234 p.

[2] Stegemöller, El., Radig, H., Hibbing, P., Wingate, J., Sapienza, C. Effects of singing on voice, respiratory control and quality of life in persons with Parkinson's disease. Disability and Rehabilitation, 2016. 39 (6): 594. DO I: $10.3109 / 09638288.2016 .1152610$

[3] Stegemöller, El., Hibbing, P., Radig, H., Wingate, J. Therapeutic singing as an early intervention for swallowing in persons with Parkinson's disease. Complementary Therapies in Medicine, 2017. 31: 127-133. DOI: 10.1016/ j.ctim.2017.03.002

[4] Barnish, J., Atkinson, R., Barran, S., Barnish, M. Potential Benefit of Singing for People with Parkinson's Disease: A Systematic Review. Journal of Parkinson's Disease, 2016. 6 (3): 473-484. DOI : 10.3233/JPD-160837.

[5] Nardone, G., Watzlawick, P. The art of change : Brief Strategic Therapy: translated from Italian. 2006. Moscow: Publishing House of the Psychotherapy Institute. 192 p. 